\title{
Comparative Study on Output Game models of Multiple Oligopolistic Manufacturers
}

\author{
Wang Guanhui ${ }^{1}$ \\ ${ }^{1}$ International business school, Tianjin Foreign Studies University, Tianjin 300204
}

\begin{abstract}
This paper compares two types of models from the aspects of function selection, hypothesis parameters and modeling basis, and further constructs two composite comparative yield game models to further analyze the cooperation and competition of node enterprises in the supply chain. Based on the output game model of the three-level supply chain, a kind of control system which makes the decision of distributors and retailers chaotic is constructed, and the simulation analysis and supply chain application interpretation are given.
\end{abstract}

\section{Introduction}

With the advent of economic globalization and knowledge economy, supply chain management is widely used in manufacturing management. It is based on the demand of the customers and the market that the manufacturers develop products, purchase raw materials which are then processed into finished goods and sold to customers. With the further division of labor, the enterprises in the supply chain are more and more specialized in certain subsections along the product life cycle. There is a constant and complicated game among enterprises for the allocation of productivity and profit.

More and more specialists and scholars are now interested in the study of the enterprises' game $^{[1-11]}$, However, this paper approaches the same issue from the angle of nonlinear dynamics, which is a new attempt. In the next section, we construct a discrete dynamical model of supply chain under the circumstances of information asymmetry and analyze its dynamic behavior in light of nonlinear dynamics.

\section{Model Constructions and Analysis}

\subsection{Nomenclature and Model Construction}

Different importance is assigned to the three traditional players along the supply chain, with the manufacturers playing a dominant role and as the focus of the study.

The following is a list of notations that will be used throughout the paper.

Nomenclature 2.2.1 $q_{i, t}$ is the production decision making of enterprise $i$ in period $t$.

Nomenclature 2.2.2 $Q$ is the total output of all the manufacturers.

$$
\begin{aligned}
& Q=q_{1}+q_{2}+\cdots, \cdots+q_{i} \\
& P(t)=\alpha+\beta Q(t)-\gamma Q(t)^{2}
\end{aligned}
$$

$P_{i, t}$ is the nonlinear inverse demand function for enterprise $i$ in period $t$.

$$
C_{i, t}=a_{i}+b_{i} q_{i, t}+c_{i} q_{i, t}^{2}
$$

$C_{i, t}$ is the cost function for enterprise $i$ in period $t$.

$\pi_{i, t}=P_{i, t} q_{i, t}-C_{i, t}$

$\pi_{i, t}$ is the profit of enterprise $i$ in period $t$.

Due to the bounded rationality and information asymmetry among the enterprises along the supply chain, when it comes to production decision making, the enterprises tend to increase the output until the maximum margin profit is attained. Therefore,

$\partial \pi_{i, t} / \partial q_{i, t}=-\gamma_{i} Q(t)^{2}+\beta Q(t)+\beta q_{i, t} Q(t)-2 \gamma q_{i, t} Q(t)-2 c_{i} q_{i, t} Q(t)+\alpha_{i}-b_{i}$

The model can be constructed as follow:

$$
q_{i, t+1}=q_{i, t}+k_{i} q_{i, t} \partial \pi_{i, t} / \partial q_{i, t}
$$

where $k_{i}$ is the output adjustment coefficient for enterprise $i$.

Then, the dynamic adjustment of the output of the upstream enterprises - the distributors - can be written as follow:

wgh@tjfsu.edu.cn 


$$
\begin{aligned}
& q_{1, t+1}=q_{1, t}+k_{1} q_{1, t}\left(-\gamma_{1} Q(t)^{2}+\beta Q(t)+\beta q_{1, t} Q(t)-2 \gamma q_{1, t} Q(t)\right. \\
& \left.-2 c_{1} q_{1, t} Q(t)+\alpha_{1}-b_{1}\right)
\end{aligned}
$$

Likewise, the dynamic adjustment of the output of enterprise $i$ can be written as follow:

$$
\begin{aligned}
& q_{i, t+1}=q_{i, t}+k_{i} q_{i, t}\left(-\gamma_{i} Q(t)^{2}+\beta Q(t)+\beta q_{i, t} Q(t)-2 \gamma q_{i, t} Q(t)\right. \\
& \left.-2 c_{i} q_{i, t} Q(t)+\alpha_{i}-b_{i}\right)
\end{aligned}
$$

Hence, the output game model can be represented by an n-dimensional nonlinear map.

$$
\left\{\begin{array}{l}
x_{1}^{\prime}=x_{1}+k_{1} x_{1}\left[-\gamma_{1} Q(t)^{2}+\beta Q(t)+\beta q_{1, t} Q(t)-2 \gamma q_{1, t} Q(t)-2 c_{1} q_{1, t} Q(t)+\alpha_{1}-b_{1}\right] \\
x_{2}^{\prime}=x_{2}+k_{2} x_{2}\left[-\gamma_{2} Q(t)^{2}+\beta Q(t)+\beta q_{2, t} Q(t)-2 \gamma q_{2, t} Q(t)-2 c_{2} q_{2, t} Q(t)+\alpha_{2}-b_{2}\right] \\
\vdots \\
x_{i}^{\prime}=x_{i}+k_{i} x_{i}\left[-\gamma_{i} Q(t)^{2}+\beta Q(t)+\beta q_{i, t} Q(t)-2 \gamma q_{i, t} Q(t)-2 c_{i} q_{i, t} Q(t)+\alpha_{i}-b_{i}\right] \\
\vdots \\
x_{n}^{\prime}=x_{n}+k_{n} x_{n}\left[-\gamma_{n} Q(t)^{2}+\beta Q(t)+\beta q_{n, t} Q(t)-2 \gamma q_{n, t} Q(t)-2 c_{n} q_{n, t} Q(t)+\alpha_{n}-b_{n}\right]
\end{array}\right.
$$

We can interpret this multi-dimensional discrete dynamical system through a study on the first map. Assume the out game among three manufacturers in

$$
\begin{aligned}
& \text { Let } q_{1, t}=x, \quad q_{2, t}=y, \quad q_{3, t}=z, q_{1, t+1}=x^{\prime}, q_{2, t+1}=y^{\prime}, q_{3, t+1}=z^{\prime} \\
& \left\{\begin{array}{l}
x^{\prime}=x+k_{1} x\left[(\beta-3 \gamma) x^{2}-\gamma y^{2}-\gamma z^{2}+(\beta-4 \gamma) x y+(\beta-4 \gamma) x z-2 \gamma y z\right. \\
\left.+\beta(x+y+z)-2 c_{1} x+\alpha-b_{1}\right] \\
y^{\prime}=y+k_{2} y\left[(\beta-3 \gamma) y^{2}-\gamma x^{2}-\gamma z^{2}+(\beta-4 \gamma) y z+(\beta-4 \gamma) x y-2 \gamma x z\right. \\
\left.+\beta(x+y+z)-2 c_{2} y+\alpha-b_{2}\right] \\
z^{\prime}=z+k_{3} z\left[(\beta-3 \gamma) z^{2}-\gamma x^{2}-\gamma y^{2}+(\beta-4 \gamma) y z+(\beta-4 \gamma) x z-2 \gamma x y\right. \\
\left.+\beta(x+y+z)-2 c_{3} z+\alpha-b_{3}\right]
\end{array}\right.
\end{aligned}
$$

\subsection{Construction and Complex Dynamics Analysis of Compound Comparison Yield Game Model}

In the model, if the oligopolistic producer represented by $\mathrm{X}$ is to make a production decision, it should also consider the order quantity of one distributor of the lower supply chain are taken into consideration. Then a nonlinear dynamic model is constructed as follows: level (if considering the modeling and analysis methods of multiple distributors), the oligopolistic producer is at A stronger position requires more consideration in the game and is closer to reality. Under this condition, a four-dimensional model of the composite yield game of a tri-oligomer producer is constructed:

$$
\left\{\begin{array}{l}
x^{\prime}=x+k_{1}(x+w)\left[(\beta-3 \gamma) x^{2}-\gamma y^{2}-\gamma z^{2}+(\beta-4 \gamma) x y+(\beta-4 \gamma) x z\right. \\
\left.-2 \gamma y z+\beta(x+y+z)-2 c_{1} x+\alpha-b_{1}\right] \\
y^{\prime}=y+k_{2} y\left[(\beta-3 \gamma) y^{2}-\gamma x^{2}-\gamma z^{2}+(\beta-4 \gamma) y z+(\beta-4 \gamma) x y-2 \gamma x z\right. \\
\left.+\beta(x+y+z)-2 c_{2} y+\alpha-b_{2}\right] \\
z^{\prime}=z+k_{3} z\left[(\beta-3 \gamma) z^{2}-\gamma x^{2}-\gamma y^{2}+(\beta-4 \gamma) y z+(\beta-4 \gamma) x z-2 \gamma x y\right. \\
\left.+\beta(x+y+z)-2 c_{3} z+\alpha-b_{3}\right] \\
w^{\prime}=w+k_{4} w\left[-3 \gamma w^{2}+2\left(\beta-c_{4}\right) w+\alpha-b_{4}\right]
\end{array}\right.
$$


Obviously, the solution of the algebraic equations does not depend on the parameters $k_{1}, k_{2}, \ldots, k_{n}$, and in the yield game dynamics model, the parameters $\alpha, \beta, \gamma, a_{i}, b_{i}, c_{i}$ are relatively determined, and the yield adjustment coefficients $k_{1}, k_{2}, k_{3}$ are variable. In order to facilitate comparison and research, the parameters are fixed as follows:

$$
\begin{gathered}
\alpha=5, \quad \beta=0.5, \quad \gamma_{=1}, \quad b_{1=0.5}, \quad c_{1}=0.1, \quad b_{2}=0.4, \quad c_{2} \\
=0.2, \quad b_{3}=0.3, \quad c_{3=0.3}, \quad c_{4=0.3}, \quad b_{4}=0.4 .
\end{gathered}
$$

Then the algebraic equations become:

$$
\left\{\begin{array}{l}
x^{\prime}=x+k_{1}(x+w)\left[-2.5 x^{2}-y^{2}-z^{2}-3.5 x y-3.5 x z-2 y z+0.5(x+y+z)-0.2 x+4.5\right] \\
y^{\prime}=y+k_{2} y\left[-2.5 y^{2}-x^{2}-z^{2}-3.5 y z-3.5 x y-2 x z+0.5(x+y+z)-0.4 y+4.6\right] \\
z^{\prime}=z+k_{3} z\left[-2.5 z^{2}-x^{2}-y^{2}-3.5 y z-3.5 x z-2 x y+0.5(x+y+z)-0.6 z+4.7\right] \\
w^{\prime}=w+k_{4} w\left[-3 w^{2}+0.4 w+4.6\right]
\end{array}\right.
$$

When manufacturer $\mathrm{X}$ makes the $\mathrm{t}+1$ production decision, it needs to consider the output of all oligarchs in the $\mathrm{t}$ period, as well as the order quantity of distributors in the $t$ period. We assume that producer $\mathrm{X}$ is more among all oligopolistic producers. An oligarch of discourse power, studying the complexity of making
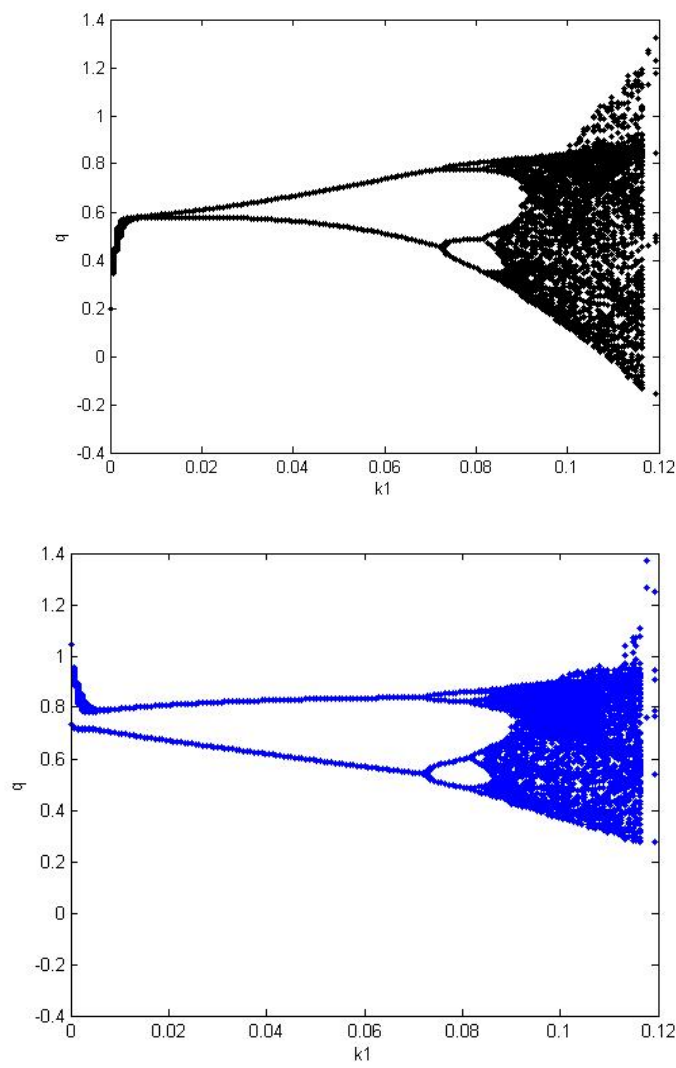

production decisions when he considers these factors. Fixed $k_{2}=0.03, k_{3}=0.02$, and adjust the production speed coefficient $k_{1}$ to observe the state effect on the production decision, as shown in Figure 1-6.
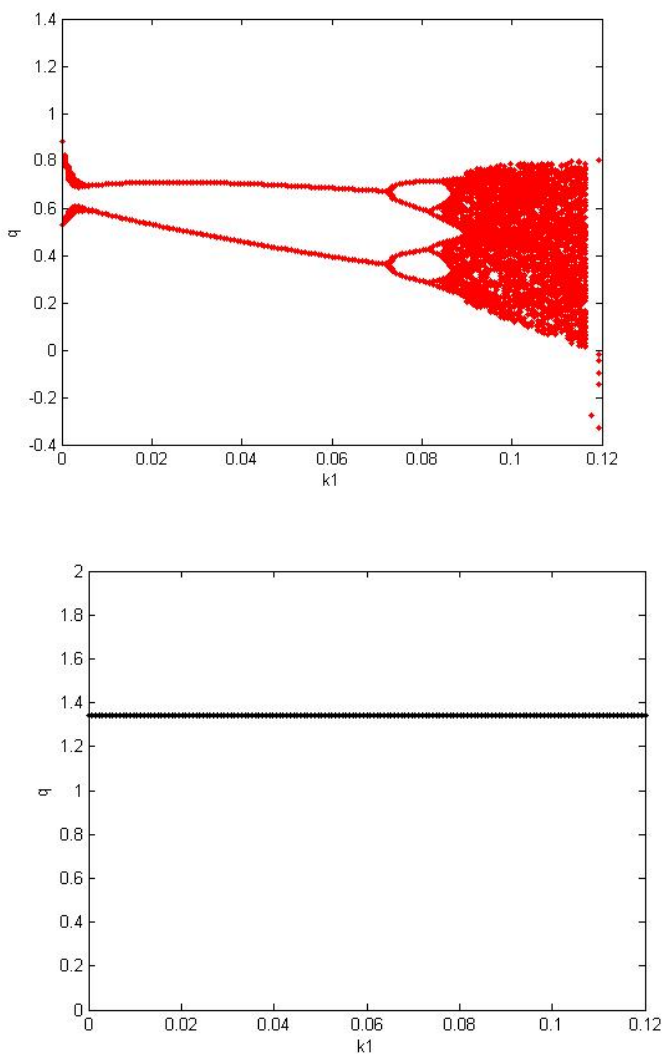

Figure 1-6(a) bifurcation diagram of production of manufacturer at $k_{2}=0.03 \quad k_{3}=0.02$

In Figure 1-6, the production decision of producer $\mathrm{X}$ is stable in the interval of $k_{1} \in(0,0.0725)$. However, as $k_{1}$ increases, the first branching occurs at $k_{1}=0.0725$

$k_{1}=0.0825$

$k_{1}=0.0848$ can be concluded that when the manufacturer additionally considers the order quantity of the distributor, the production decision will enter the chaotic state more quickly, and the stability interval is greatly reduced. The decision function of producer $\mathrm{Y}$ and producer $\mathrm{Z}$ in system 1-7 does not take into account the influence of the distributor's order quantity, but it can be seen from the simulation results that the production division diagram of producer $\mathrm{Y}$ and producer $\mathrm{Z}$ and the division of producer $\mathrm{X}$ The bifurcation point of the map is consistent with the 
trend of the double period. This shows that both producer $\mathrm{Y}$ and producer $\mathrm{Z}$ adopt a follow-up strategy, which, like the conclusion of the "smart pig game", will change according to the decision of producer $\mathrm{X}$, which has the least risk.

\section{Conclusion}

In this paper, the game models of compound comparative production are constructed, and the complex dynamic analysis is done. Compared with the data of the previous game model, we can draw a conclusion: When a producer makes a production decision, if he considers the demand across levels. he may lose the stability of the decision more quickly. which puts forward another solution to alleviate the bullwhip effect.

\section{References}

1. Hosseini, Seyedmohsen; Ivanov, Dmitry, Bayesian networks for supply chain risk, resilience and ripple effect analysis: A literature review. Expert systems with applications, Volume 161, 113649, 2020

2. Ayoughi, Providing an Integrated Multi-Objective Model for Closed-Loop Supply Chainunder Fuzzy Conditions with Upgral Approach, INTERNATIONAL JOURNAL OF NONLINEAR ANALYSIS AND APPLICATIONS, Volume 11, Issue 1, 2020, Pages 107-136

3. Tliche, Youssef, An improved forecasting approach to reduce inventory levels in decentralizedsupply chains, EUROPEAN JOURNAL OF OPERATIONAL RESEARCH, Volume 287, Issue 2, 2020, Pages 511-527

4. Yugang $\mathrm{Yu}$, George Q. Huang, Liang Liang, Stackelberg game-theoretic model for optimizing advertising, pricing and inventory policies in vendor managed inventory (VMI) production supply chains Original Research Article, Computers \& Industrial Engineering, Volume 57, Issue 1, August 2009, Pages 368-382

5. H. Brian Hwarng, Na Xie, Understanding supply chain dynamics: A chaos perspective Original Research Article, European Journal of Operational Research, Volume 184, Issue 3, 1 February 2008, Pages 1163-1178

6. Jose B. Cruz Jr., Raymond R. Tan, Alvin B. Culaba, Jo-Anne Ballacillo, A dynamic input-output model for nascent bioenergy supply chains Original Research Article, Applied Energy, Volume 86, Supplement 1, November 2009, Pages S86-S94

7. Hong-jun PENG, Mei-hua ZHOU, Man-zhi LIU, Yu ZHANG, Yan-bo HUANG, A dynamic optimization model of an integrated coal supply chain system and its application, Original Research Article, Mining Science and Technology (China), Volume 19, Issue 6, November 2009, Pages 842-846

8. Ni DB, Li KW, Tang XO. Complexity of ordering dynamics under pure revenue-sharing contracts[C].
In: 2006 IEEE International Conference on Systems, Man, and Cybernetics, Vols 1-6, Proceedings. Taipei; 2006:936-941.

9. Zhang L, Li YJ, Xu YQ. Chaos synchronization of bullwhip effect in a supply chain[C]. In: Lan H, ed. Proceedings of the 2006 International Conference on Management Science \& Engineering (13th), Vols 1-3. Lille; 2006:557-560.

10. Wu Y, Zhang DZ. Demand fluctuation and chaotic behaviour by interaction between customers and suppliers[J]. International Journal of Production Economics. 2007, 107(1): 250-259.

11. Hwarng HB, Xie N. Understanding supply chain dynamics: A chaos perspective[J]. European Journal of Operational Research. 2008, 184(3): 1163-1178. 